\title{
Systematic Construction of Finite State Automata Using VLSI Spiking Neurons
}

\author{
Emre Neftci ${ }^{1}$, Jonathan Binas ${ }^{1}$, Elisabetta Chicca ${ }^{2}$, Giacomo Indiveri ${ }^{1}$, \\ and Rodney Douglas ${ }^{1}$ \\ 1 Insitute of Neuroinformatics, University of Zurich and ETH Zurich, \\ Wintherthurerstrasse 190, 8057 Zurich \\ 2 Cognitive Interaction Center of Excellence (CITEC), University of Bielefeld, \\ Universitätstrasse 21-23, Bielefeld, Germany
}

Spiking neural networks implemented using electronic Very Large Scale Integration (VLSI) circuits are promising information processing architectures for carrying out complex cognitive tasks in real-world applications. These circuits are developed using standard silicon technologies, and exploit the analog properties of transistors to emulate the phenomena underlying the computations and the communication in the brain. Neuromorphic multi-neuron systems can provide a low-power and scalable information processing technology, that is optimally suited for advanced and future VLSI processes [1].

However, because the relationship between function and neural architecture is still not understood, their configuration to perform target state-dependent computations is a challenging task. Moreover, due to the non-linear relationship between the chip parameters and those of theoretical neuron models, the configuration of the hardware neurons to match the target behavior is not straightforward.

We introduce a procedure to configure real-time VLSI spiking neural networks, which solves these two problems by mapping the computations of classical Finite State Automata (FSA) onto the neural circuit. The procedure systematically constructs a neural architecture based on a class of biologically inspired, stereotypical circuits expressing the Soft Winner-Take-All (sWTA) function [2]. Using a parameter translation technique [3], the procedure systematically maps the key characteristics of the sWTA network onto its electronic counterpart.

Fig. 1a illustrates the neural FSA architecture consisting of three sWTA networks (gray-shaded rectangles). The state is maintained in working memory in the form of persistent activity, using two recurrently coupled sWTA networks (horizontal and vertical rectangles). These are coupled such that there are as many distinct populations able to maintain persistent activity as possible states in the FSA. A third sWTA network (diagonally shaded) mediates the transitions between the states, and each of its populations are receptive to one external input and one state. Using a mean-field formalism, the parameters of this sWTA are computed such that only a single population activates when an input is provided 4]. The neural FSA is constructed by connecting the output of each transition population to the target states, according to the state diagram.

Fig. 1b] shows the response of a neuromorphic chip implementing the neural FSA during an input string consisting of five X's, provided at random intervals (blue shadings). The neural system responds in a state-dependent manner, in that its activity is conditional on the input and the previous state.

In principle, this procedure can be applied to generate the circuitry for implementing automata of almost any complexity [2]. This achievement is an 
(i)

(ii)

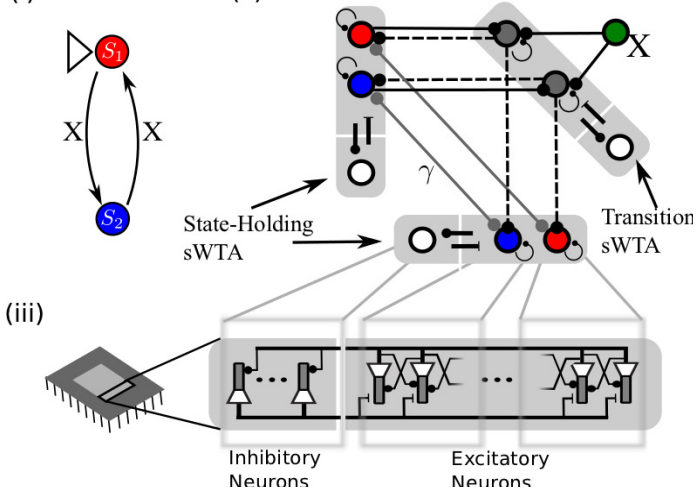

a Neural Finite State Automaton
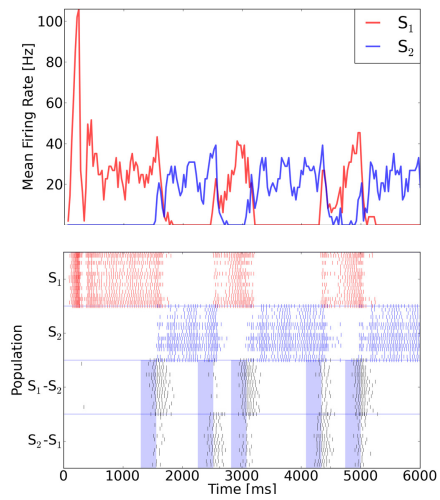

b VLSI chip response

Fig. 1. (国) (i) State diagram where circles indicate states and arrows the transitions between them. The white triangle specifies the initial state. For this automaton, the active state flips between $S_{1}$ and $S_{2}$ in response to X. (ii) The neural circuit implementing the automaton in (i). (iii) The multi-neuron chips used in the neuromorphic setup feature low-power integrate-and-fire neurons with dynamic synapses. Each population of a sWTA network (circle) is represented in hardware by 16 recurrently coupled neurons, which compete against the other populations via global inhibition. (b) (top) Average firing rate one state-holding sWTA. The inputs were provided at arbitrary intervals to the transition neurons (blue shadings). (b) (bottom) Raster plot of the activity in the stateholding elements and the transitions networks ( $S_{1}$ to $S_{2}$ and $S_{2}$ to $\left.S_{1}\right)$. Consistently with the state diagram, the neural system reliably flips its state at each presentation of $\mathrm{X}$.

important milestone towards building generic-purpose neuromorphic systems that are able to interact with unconstrained real-world environments in space- and power-constrained applications, such as in robotic or mobile hand-held devices. In future work, we will couple these neuromorphic hardware with event-based vision sensors to achieve sophisticated sensorimotor tasks.

Acknowledgements. This work was supported by the EU ERC Grant "neuroP" (257219), the EU ICT Grant "SCANDLE" (231168) and the Excellence Cluster 227 (CITEC, Bielefeld University).

\section{References}

[1] Indiveri, G., Linares-Barranco, B., Hamilton, T., van Schaik, A., EtienneCummings, R., et al.: Neuromorphic silicon neuron circuits. Frontiers in Neuroscience 5, 1-23 (2011)

[2] Rutishauser, U., Douglas, R.: State-dependent computation using coupled recurrent networks. Neural Computation 21, 478-509 (2009)

[3] Neftci, E., Chicca, E., Indiveri, G., Douglas, R.: A systematic method for configuring VLSI networks of spiking neurons. Neural Computation 23(10), 2457-2497 (2011)

[4] Neftci, E.: Towards VLSI Spiking Neuron Assemblies as General-Purpose Processors. Ph.D. thesis, ETH Zürich (2010) 